\title{
Comment on linear data-fitting methods
}

Giorgio Gambirasio (giga@uol.com.br)

\begin{abstract}
Fitting the best straight line through experimental points is a common practice performed with a standard methodology. However, as it is commented about on this paper, there is more than one useful method to compute the best line.
\end{abstract}

Keywords: data fitting, least square, best line.

\section{Introduction}

Running the best straight line thorough scattered points is an old and noble contribution of Mathematics to experimental sciences. There are many possible approaches and methods to obtain the best line. Each approach gives its own "best" line, different from the lines obtained using other approaches.

The oldest and to-days' most popular method (which even attracted the attention of mathematicians like Gauss and Lagrange) runs as follows:

Consider the vertical distance from each experimental point to the yet unknown best straight line as being the error of that point. The errors of all points are squared and summed up to a total-squared-error $\mathrm{E}^{2}$. The best straight line is the line that minimizes $\mathrm{E}^{2}$. A simple and direct approach to the problem!

Advantages of this approach:

1) squaring individual errors avoids mutual cancellation when positive and negative errors are summed;

2) the squaring of individual errors may be explicitly made (it gives polynomials), then $E^{2}$ is the sum of as many polynomials as there are experimental points;

3) the straight line is described by two parameters, the two parameters are chosen such as to minimize $\mathrm{E}^{2}$ :

4) doing such minimization is an easy job: compute the partial derivatives of $E^{2}$ with respect to each parameter, get a system of two linear equations, solve such a system and obtain the parameters.

And the best line is found!

The method that we just finished describing will be considered a basic item for this study, where different criteria for finding the best line will be reviewed. Our study uses elementary mathematics only, with no mention of other things like regression and correlation coefficient. 
But it must be noted that the approach of "squaring before summing" suffers from a serious disadvantage: since the individual errors are squared, each contribution to the total error is also squared. Points far away from the line then give an exaggerated contribution to $\mathrm{E}^{2}$ because they attract the line towards them too much.

\section{Notation, auxiliary programs, etc.}

In this overview of different criteria for getting the best line through the experimental points, we will not actually perform the computations leading to the best line parameters. We shall leave the purely numerical job of minimization to an applet freely provided by Wolfram Alpha (https://www.wolframalpha.com/input/?i=minimize).

Other useful calculators are also available on the Web. All these devices consider line parameters as unknowns, labeling them $\mathrm{x}$ and $\mathrm{y}$. It would also be confusing to label $\mathrm{x}$ and $\mathrm{y}$ the coordinate axes where the experimental points are located, and these axes will instead be labeled $\mathbf{s}$ (horizontal axis) and $\mathbf{t}$ (vertical axis).

In conclusion, the best straight line will now be written $\mathbf{t}=\mathbf{x} \mathbf{s}+\mathbf{y}$.

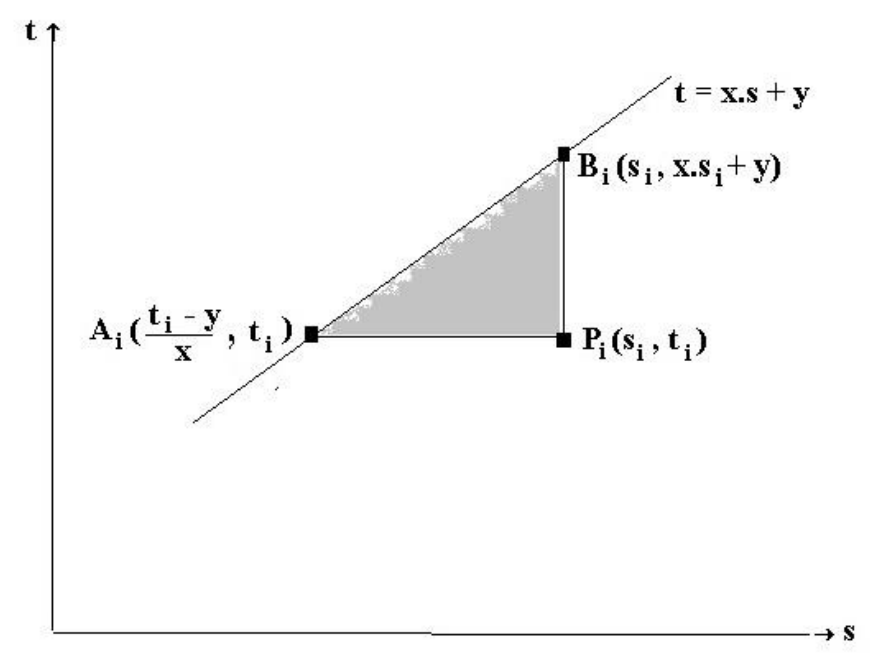

Fig. $1-$ Generic position of an experimental point $\mathrm{P}_{\mathrm{i}}$ in relation to the best straight line $\mathrm{t}=\mathrm{x} \mathrm{s}+\mathrm{y}$.

Plotted experimental points are $\mathrm{P}_{\mathrm{i}}\left(\mathrm{s}_{\mathrm{i}}, \mathrm{t}_{\mathrm{i}}\right)$. Subscript $\mathrm{i}$, either explicitly shown (as in $\left.\sum_{\mathrm{i}}\right)$ or not (as in $\sum$ ), will always go from $i=1$ to $i=n$.

Some numerical examples are built around the following set of $n=6$ scattered experimental points shown on Table I. 
Table I - Experimental points and related sums to be used in an example

\begin{tabular}{|c|c|c|}
\hline $\mathbf{S}_{\mathbf{i}}$ & $\mathbf{t}_{\mathbf{i}}$ & Sums \\
\hline 1 & 5 & $\Sigma\left(\mathrm{s}_{\mathrm{i}}\right)=21$ \\
\hline 2 & 6 & $\Sigma\left(\mathrm{t}_{\mathrm{i}}\right)=41$ \\
\hline 3 & 4 & $\Sigma\left(\mathrm{s}_{\mathrm{i}} \mathrm{t}_{\mathrm{i}}\right)=167$ \\
\hline 4 & 4 & $\Sigma\left(\mathrm{s}_{\mathrm{i}}{ }^{2}\right)=91$ \\
\hline 5 & 10 & $\Sigma\left(\mathrm{t}_{\mathrm{i}}{ }^{2}\right)=337$ \\
\hline 6 & 12 & $\Sigma(1)=\mathrm{n}=6$ \\
\hline
\end{tabular}

\section{The vertical error VE approach}

We will now give attention to the oldest and most used approach, in which the sum of squared vertical (along $t$ axis) distance of each experimental point $\mathrm{P}_{\mathrm{i}}\left(\mathrm{s}_{\mathrm{i}}, \mathrm{t}_{\mathrm{i}}\right)$ from the best line $\mathrm{t}=\mathrm{x} \mathrm{s}+$ $\mathrm{y}$ is taken into account as an error to be minimized. The distance is shown on Figure 1 to be equal to the length of segment $\mathrm{B}_{\mathrm{i}} \mathrm{P}_{\mathrm{i}}$.

The squared vertical error related to a generic point $\mathrm{P}_{\mathrm{i}}\left(\mathrm{s}_{\mathrm{i}}, \mathrm{t}_{\mathrm{i}}\right)$ has value $\left(\mathrm{x} \mathrm{s} \mathrm{s}_{\mathrm{i}}+\mathrm{y}-\mathrm{t}_{\mathrm{i}}\right)^{2}$, and the total square vertical error (labeled VE) is therefore:

$$
\mathrm{VE}=\sum_{\mathrm{i}}\left(\mathrm{x} \mathrm{s}_{\mathrm{i}}+\mathrm{y}-\mathrm{t}_{\mathrm{i}}\right)^{2}, \quad \mathrm{i}=1,2, . ., \mathrm{n} .
$$

Each squared error may be explicitly written as a polynomial

$$
\left(x s_{i}+y-t_{i}\right)^{2}=x^{2} s_{i}^{2}+y^{2}+t_{i}^{2}+2 x y s_{i}-2 x s_{i} t_{i}-2 y t_{i} .
$$

After summing and collecting terms one gets the general expression of the total vertical square error:

$$
V E=x^{2} \Sigma\left(s_{i}^{2}\right)+n y^{2}+\Sigma\left(t_{i}^{2}\right)+2 x y \Sigma\left(s_{i}\right)-2 x \Sigma\left(s_{i} t_{i}\right)-2 y \Sigma\left(t_{i}\right)
$$

Remember that the short-hand symbol $\Sigma$ means summing all values from $\mathrm{i}=1$ to $\mathrm{i}=\mathrm{n}$. For the numerical example of Table I, all $\Sigma$ sums are shown in the last column of the table.

Using values of Table I, VE becomes

$$
V E=91 x^{2}+6 y^{2}+337+42 x y-334 x-82 y
$$

It is this the function which, when minimized, gives the values of $\mathrm{x}$ and $\mathrm{y}$ and therefore the best line $\mathrm{t}=\mathrm{xs}+\mathrm{y}$. 
Of course, in this case of vertical errors, one can find many calculators on the Web which quickly give $\mathrm{x}$ and $\mathrm{y}$ and more information also. However, this bounty of calculators is restricted to the case of vertical errors. If other approaches were preferred, a minimizing applet like the one offered by Wolfram Alfa becomes the useful tool.

For the sake of uniformity, we shall use the Wolfram applet. It gives the minimum of VE equal to 25.3 at $\mathrm{x}=1.34$ and $\mathrm{y}=2.13$. Then, the best line for the example of Table I, according to vertical error criterium, is $\mathrm{t}=1.34 \mathrm{~s}+2.13$.

Rewritten in Wolfram language, VE becomes

$$
\mathrm{VE}=91 \mathrm{x}^{\wedge} 2+6 \mathrm{y}^{\wedge} 2+337+42 x y-334 x-82 \mathrm{y} .
$$

The reader can just copy and paste it into the applet.

Besides being in almost exclusive use since old times, VE has one more characteristic, as it will be seen in the following: it appears as a factor in the computation of other approaches to the problem of line fitting.

\section{The horizontal error HE approach}

But why the vertical error approach only should be deemed important? We live in an age in which neglected minorities furiously claim their place in the sun. The social discrimination suffered by horizontal error is particularly shameful because horizontal errors do not sum up to a minority, they exist in the same quantity as the vertical ones. As Figure 1 clearly shows, each experimental point generates one vertical error and one horizontal error also. So, let us go and correct such social injustice.

According to Figure 1, the horizontal distance of generic point $\mathrm{P}_{\mathrm{i}}$ to best line equals the length of segment $\mathrm{A}_{\mathrm{i}} \mathrm{P}_{\mathrm{i}}$. The squared horizontal error related to a generic point $\mathrm{P}_{\mathrm{i}}\left(\mathrm{s}_{\mathrm{i}}, \mathrm{t}_{\mathrm{i}}\right)$ has value $\left[\left(\mathrm{t}_{\mathrm{i}}-\right.\right.$ y) $\left.\mathrm{x}^{-1}-\mathrm{si}_{\mathrm{i}}\right]^{2}$, and the total square horizontal error (labeled HE) is therefore:

$$
\mathrm{HE}=\Sigma\left[\left(\mathrm{t}_{\mathrm{i}}-\mathrm{y}\right) \mathrm{x}^{-1}-\mathrm{s}_{\mathrm{i}}\right]^{2}=\Sigma \mathrm{x}^{-2}\left(\mathrm{t}_{\mathrm{i}}-\mathrm{y}-\mathrm{x} \mathrm{s}_{\mathrm{i}}\right)^{2}=\mathrm{x}^{-2} \Sigma\left(\mathrm{t}_{\mathrm{i}}-\mathrm{y}-\mathrm{x} \mathrm{s}_{\mathrm{i}}\right)^{2} .
$$

Always remember that $\Sigma$ means summing from $\mathrm{i}=1$ to $\mathrm{i}=\mathrm{n}$.

We can immediately write $\mathrm{HE}=\mathrm{x}^{-2} \mathrm{VE}$, then

$$
\min (H E)=\min \left(x^{-2} V E\right)
$$

[Attention! $\min \left(\mathrm{x}^{-2} \mathrm{VE}\right)$ in NOT equal to $\mathrm{x}^{-2} \min (\mathrm{VE})$ ]

This means that once the reader has minimized VE with the Wolfram applet, he/she may keep the page open and add factor $x^{\wedge} 2$ to the already written VE. Then minimize, and HE results will appear.

For the case of Table I, the results are: minimum of HE is equal to 7.8 at $\mathrm{x}=2.42$ and $\mathrm{y}=$ -1.63 . Then the best line, according to horizontal error criterium, is $t=2.42 \mathrm{~s}-1.63$.

The new line is not just a copy of the line given by VE, it is not a piece of redundant information! It is really new information, sometimes a better one. And obviously it is equally entitled to be the best line. 


\section{Vertical and horizontal errors simultaneously (HYE)}

VE and HE usually give different lines, one of them seeming more suitable than the other to represent the best fit. Which one looks better is not known beforehand, it depends on how the cloud of experimental points is shaped. Then, why not try to use both approaches at the same time, in order to get a kind of average or mixed best line?

A simple solution to do this is the direct summing of VE with HE to get a new total square error HYE:

$$
H Y E=V E+H E=V E\left(1+x^{-2}\right)
$$

Notice that both VE and HE are polynomials and may be simply summed. It is now HYE that should be minimized to get a new best line. This line comes from the joint contribution of vertical and horizontal errors.

But this is not all. With reference to Figure 1, it may be seen that getting HYE means summing all the squared segments $A_{i} P_{i}$ with the corresponding squared segments $\mathrm{B}_{i} \mathrm{P}_{\mathrm{i}}$. Since $\mathrm{A}_{i} \mathrm{~B}_{i} \mathrm{P}_{i}$ is a right triangle, by Pythagoras's theorem that sum equals the sum of all squared hypotenuses $A_{i} B_{i}$. In other words, HYE is the sum of the square of all individual hypotenuses of all right triangles formed by experimental points. The inclusion of both errors (vertical and horizontal ones) in the quantity to be minimized actually results in a new approach: minimization of hypotenuse's errors.

For the case of the numerical example (Table I), pasting

$$
\operatorname{VE}\left(1+x^{\wedge}-2\right)=\left(91 x^{\wedge} 2+6 y^{\wedge} 2+337+42 x y-334 x-82 y\right)\left(1+x^{\wedge}-2\right)
$$

into the Wolfram applet gives the minimum of HYE equal to 36.7 and the corresponding line as $\mathrm{t}=1.61 \mathrm{~s}+1.21$.

\section{Graphical comparison of the approaches}

Experimental points in Table I have been used to compute the straight lines according to approaches VE, HE, and HYE. Resulting lines' equations are:

1) by vertical errors approach VE: $t=1.345 \mathrm{~s}+2.13$;

2) by horizontal errors approach HE: $t=2.42 \mathrm{~s}-1.63$;

3) by hypotenuse's errors approach HYE: $\mathrm{t}=1.61 \mathrm{~s}+1.21$.

Figure 2 shows the three lines plotted on the same graph. Experimental points are also shown on the graph (by crosses +). In this case, the line given by VE looks like a better line than the line by HE. And the line given by HYE seems to have an even better fit. 


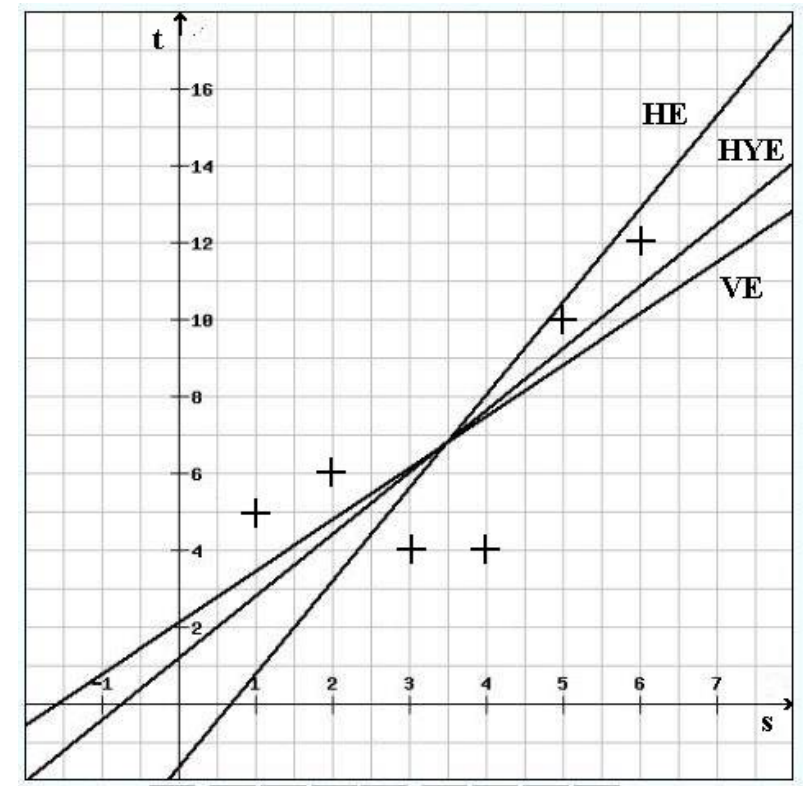

Figure 2 - Lines computed by vertical (VE), horizontal (HE), and hypotenuse (HYE) approaches from experimental data of Table I, all plotted on the same diagram. The positions of experimental points are indicated by crosses $(+)$.

\section{The problem of outliers}

As it was mentioned before, squaring the errors before summing them exaggerates the influence of points far away from the line. These points, called outliers, tend to strongly attract the line towards them and may seriously distort the final result.

In order to assess how each of the three approaches behaves when confronted with an outlier, two examples have been prepared.

Table II - Data for testing with the first outlier, located at $(4,20)$

\begin{tabular}{|c|c|c|}
\hline $\mathbf{S}_{\mathbf{i}}$ & $\mathbf{t}_{\mathbf{i}}$ & Sums \\
\hline 1 & 1 & $\Sigma\left(\mathrm{s}_{\mathrm{i}}\right)=21$ \\
\hline 2 & 2 & $\Sigma\left(\mathrm{t}_{\mathrm{i}}\right)=37$ \\
\hline 3 & 3 & $\Sigma\left(\mathrm{s}_{\mathrm{i}} \mathrm{t}_{\mathrm{i}}\right)=155$ \\
\hline 4 & 20 & $\Sigma\left(\mathrm{s}_{\mathrm{i}}{ }^{2}\right)=91$ \\
\hline 5 & 5 & $\Sigma\left(\mathrm{t}_{\mathrm{i}}{ }^{2}\right)=475$ \\
\hline 6 & 6 & $\Sigma(1)=\mathrm{n}=6$ \\
\hline
\end{tabular}

Table II and Table III present the data, each one with six experimental points. One of the points is a strong outlier. The outlier has been put very far from the other five points on purpose so that the attraction would be very noticeable, as the following figures show. 
The other five points (without outlier) lay on the line $t=s$, that is, on a forty-five-degree straight line through the origin. But when the outlier is included, it attracts and therefore changes the line. Each approach responds differently to the outlier and the purpose of this exercise is to compare their behaviors.

The two graphs (Figure 3 and Figure 4) are plotted with the same range of axes, number of intervals, and reticule lines, for easier comparison.

For data from Table II the results are:

VE: minimum $=210 ; \mathrm{t}=1.46 \mathrm{~s}+1.07$;

$\mathrm{HE}:$ minimum $=14.9 ; \mathrm{t}=9.7 \mathrm{~s}-27.7$;

HYE: minimum $=264 ; \mathrm{t}=2.32 \mathrm{~s}-1.95$.

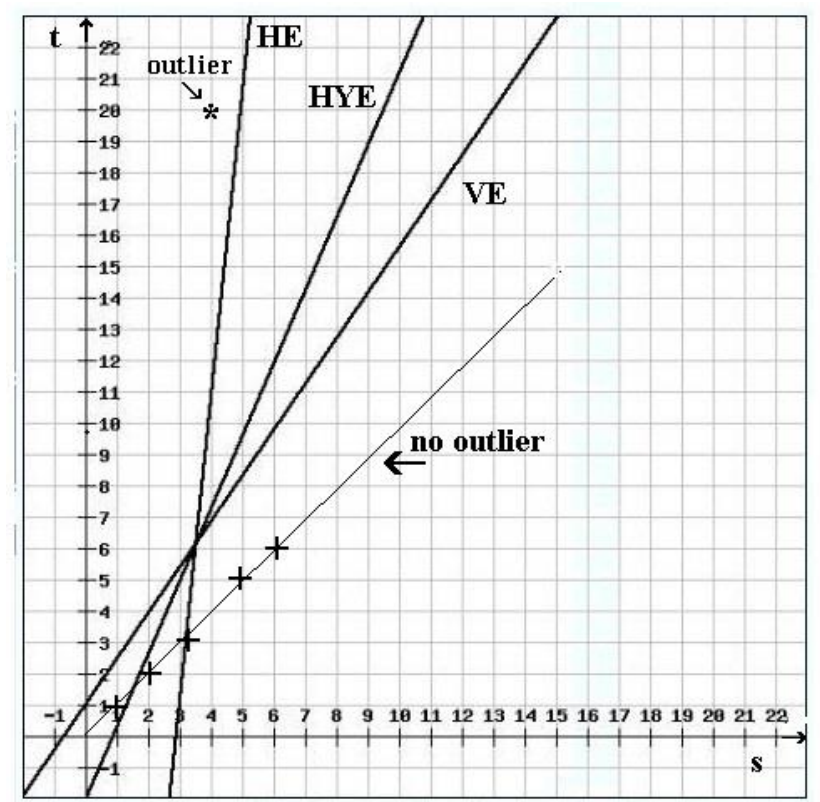

Fig. 3 - Results from applying VE, HE, and HYE to data of Table II. Experimental points are indicated by crosses $(+)$; the outlier by a star $(*)$. The line obtained when outlier is not included is labeled "no outlier".

Table III - Data for testing with the second outlier, located at $(20,4)$

\begin{tabular}{|c|c|c|}
\hline $\mathbf{s}_{\mathbf{i}}$ & $\mathbf{t}_{\mathbf{i}}$ & Sums \\
\hline 1 & 1 & $\Sigma\left(\mathrm{s}_{\mathrm{i}}\right)=37$ \\
\hline 2 & 2 & $\Sigma\left(\mathrm{t}_{\mathrm{i}}\right)=21$ \\
\hline 3 & 3 & $\Sigma\left(\mathrm{s}_{\mathrm{i}} \mathrm{t}_{\mathrm{i}}\right)=155$ \\
\hline 20 & 4 & $\Sigma\left(\mathrm{s}_{\mathrm{i}}{ }^{2}\right)=475$ \\
\hline outlier) & 5 & $\Sigma\left(\mathrm{t}_{\mathrm{i}}{ }^{2}\right)=91$ \\
\hline 5 & 6 & $\Sigma(1)=\mathrm{n}=6$ \\
\hline 6 & & \\
\hline
\end{tabular}


For data from Table III the results are:

VE: minimum $=14.9 ; \mathrm{t}=0.103 \mathrm{~s}+2.86$;

HE: minimum $=210 ; \mathrm{t}=0.686 \mathrm{~s}-0.732$;

HYE: minimum $=264 ; \mathrm{t}=0.431 \mathrm{~s}+0.84$.

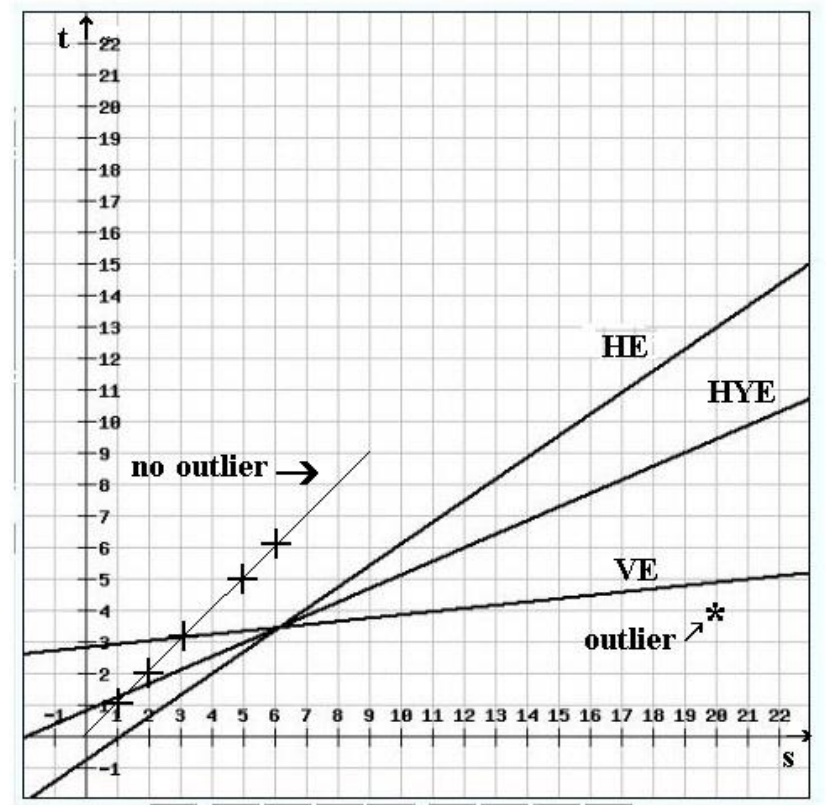

Fig. 4 - Results from applying VE, HE, and HYE to data of Table III. Experimental points are indicated by crosses $(+)$; the outlier by a star $\left(^{*}\right)$. The line obtained when outlier is not included is labeled "no outlier".

These tests show that either VE or HE may show greater sensitivity to an outlier, all depending on the distribution of experimental points. HYE, however, seems to always represent a good compromise between extreme behaviors.

\section{Conclusions}

This brief review shows that in problems of least square data-fitting it is worth looking outside of the conventional standard vertical errors' methodology. Considering hypotenuse's errors may lead to a more comprehensive methodology, including the possibility of a lesser influence from outliers. 